\title{
The relationship between insight and subjective experience in schizophrenia
}

\author{
This article was published in the following Dove Press journal: \\ Neuropsychiatric Disease and Treatment \\ 29 July 2014 \\ Number of times this article has been viewed
}

\author{
Yuki Kako \\ Koki Ito \\ Naoki Hashimoto \\ Kuniyoshi Toyoshima \\ Yusuke Shimizu \\ Nobuyuki Mitsui \\ Yutaka Fujii \\ Teruaki Tanaka \\ Ichiro Kusumi \\ Department of Psychiatry, Hokkaido \\ University Graduate School \\ of Medicine, Sapporo, Japan
}

Correspondence:Yuki Kako Department of Psychiatry, Hokkaido University Graduate School of Medicine, Kita I 5 Nishi 7, Kita-ku, Sapporo, Hokkaido 060-8638, Japan

Tel $+8 \mid$ II 716 |I6I ext 5973

Fax $+8 \mid$ II 706508 ।

Email yukikako@nifty.com
Objectives: To examine the relationship between level of insight and various subjective experiences for patients with schizophrenia.

Materials and methods: Seventy-four patients with schizophrenia who were discharged from our hospital were evaluated. The level of insight into their illness and various subjective experiences were evaluated at discharge. We used the Scale to Assess Unawareness of Mental Disorder (SUMD) for evaluation of insight. In addition, five different rating scales were used to evaluate subjective experiences: Subjective Experience of Deficits in Schizophrenia (SEDS), Subjective Well-being under Neuroleptic drug treatment Short form (SWNS), Schizophrenia Quality of Life Scale (SQLS), Beck Depression Inventory (BDI), and the Drug Attitude Inventory (DAI)-30.

Results: The SWNS and the scores for awareness of mental disorder and awareness of the social consequences of mental disorder on SUMD showed a weak positive correlation. The DAI-30 showed a significant negative correlation with most general items on SUMD and a negative correlation between the subscale scores for the awareness and attribution of past symptoms. SEDS, SWNS, SQLS, and the BDI significantly correlated with the subscale scores for awareness of current symptoms on SUMD, and weakly correlated with the subscale scores for attribution of current negative symptoms.

Conclusion: Awareness of subjective distress was related to awareness of having a mental disorder. Feeling subjective distress was related to awareness of current symptoms, as well as to the ability to attribute current negative symptoms to a mental disorder. Positive attitudes toward medication correlated with better general insight into the illness.

Keywords: schizophrenia, insight, subjective experiences, subjective deficit, subjective wellbeing, subjective quality of life

\section{Introduction}

In patients with schizophrenia, poor insight into the illness is common and is the most problematic issue in treatment. To determine the factors contributing to poor insight, its relationships with various clinical indicators have been examined. This area has seen much research, particularly since the late 1980s, after methods to quantitatively evaluate insight into mental disorders were developed. ${ }^{1-5}$ Despite this, factors impacting the development of insight have not been sufficiently identified, and further investigation from various perspectives is required.

To allow patients to become aware of their own disease, it appears necessary to obtain objective data, ie, test results indicating the existence of illness. Considering that there are no objective test findings to confirm the existence of schizophrenia that allow patients to become aware of their illness, it appears necessary to ascertain whether or not they experience subjective distress. However, subjective experience has been excessively overlooked in the symptomatic assessment of schizophrenia, because of 
the long-term emphasis on objective psychopathological evaluation and because of tenuous scientific evidence. Few studies have evaluated the relationship between the subjective experience of schizophrenia and insight. ${ }^{6-8}$

Peralta and Cuesta ${ }^{6}$ examined 118 patients with schizophrenia at the time of hospital discharge. In that study, subjective experience was evaluated using the Frankfurt Complaint Questionnaire, which contained 98 items. ${ }^{9}$ However, insight was not rated with a particular scale but with dichotomous choices of presence and absence of insight, in order to compare obtained questionnaire scores. The study found that patients with insight had significantly more awareness of subjective experiences compared with their counterparts. Kim et $\mathrm{al}^{7}$ studied 63 patients with schizophrenia (20 in the acute phase and 43 in the chronic phase) to examine the relationship between insight and subjective experience. Insight was evaluated using the Schedule for Assessment of Insight, ${ }^{3}$ whereas subjective experience was examined using an original scale. However, this previous cross-sectional study did not find any significant correlation between these two measures. Therefore, Kim et al stated that the important factors influencing insight were subjective suffering previously experienced by patients and how much such suffering was diminished by treatment. Nakaya et $\mathrm{al}^{8}$ also examined 129 inpatients with chronic-phase schizophrenia to establish the relationship between Subjective Experience of Deficits in Schizophrenia (SEDS), which was used in our study, and the question items related to insight (G12) in the Positive and Negative Syndrome Scale (PANSS); ${ }^{10}$ however, no significant correlation was observed. Such inconsistent findings may be attributed to differences in attributes of the patients studied (such as duration or stage of illness) and differences in the measures evaluated. To study the relationship between insight and subjective experience further, it is necessary to evaluate insight in a multidimensional way and to evaluate subjective experience from various perspectives.

Meanwhile, tools to quantitatively evaluate subjective experience gradually developed during the 1980s. ${ }^{11-14}$ Furthermore, since the introduction of atypical antipsychotics, subjective experiences have been examined as subjective responses to pharmacotherapy, and for assessing the efficacy of pharmacotherapy, subjective quality of life (QOL), subjective well-being, and psychological compliance indicators have been examined. In addition, subjective depressive symptoms are important subjective experiences. As a result, various concepts of subjective experiences have been proposed; however, they are broad and have yet to be properly organized. Moreover, because active patient participation in therapy is preferred, the importance of subjective experiences is expected to further increase; the same principles are also applicable for insight.

We hypothesized that the aforementioned subjective experiences are important factors affecting patients' insight into the illness. To verify this hypothesis, we used the Scale to Assess Unawareness of Mental Disorder (SUMD) ${ }^{1}$ for evaluation of insight into the illness, which covers most dimensions of insight, and for subjective experience, we used five measures with different viewpoints (subjective deficits, subjective well-being, subjective QOL, subjective depression, and attitudes toward medication). Consequently, compared with previous studies, we were better able to elucidate the relationship between these two factors.

\section{Materials and methods Subjects}

Among the patients discharged from Hokkaido University Hospital's Division of Psychiatry and Neurology, we evaluated all who met Diagnostic and Statistical Manual of Mental Disorders (DSM)-IV-TR ${ }^{15}$ criteria for schizophrenia. Patients who were hospitalized for treatment of a physical complication (eg, perioperative management) were excluded. Two research psychiatrists (YK and KI) formulated diagnoses using the DSM-IV-TR, based on direct interviews with and observation of patients. Of the 78 target subjects, three were excluded because of organic brain syndrome, mental retardation, substance abuse, psychomotor excitement, or severe thought derailment. Informed consent was obtained from the remaining 75 patients with regard to conducting an interview and answering a questionnaire. However, one patient could not sufficiently understand the contents of the questionnaire to answer it appropriately. Consequently, data from 74 patients ( 37 men and 37 women) were analyzed.

Participant attributes were as follows (mean \pm standard deviation): age, 36.0 \pm 2.3 years; age of illness onset, $26.1 \pm$ 9.5 years; duration of illness, $10.0 \pm 9.7$ years; duration of education, $13.1 \pm 2.4$ years; mean number of hospitalizations, 2.8 \pm 2.5 ; and mean total duration of hospitalization, $381 \pm 506$ days. Schizophrenia type at the time of survey was classified as follows: paranoid, $n=25$; disorganized, $n=3$; undifferentiated, $n=10$; and residual, $n=36$. Illness stage was classified as follows: recovery phase after the first psychotic episode, $n=26$; acute phase of relapse, $n=1$; recovery phase after relapse, $n=44$; and chronic phase, $n=3$. The mean score on $\mathrm{PANSS}^{10}$ for evaluation of symptom severity was 63.5 \pm 16.2 . The mean score on the Global Assessment of Functioning scale ${ }^{16}$ for evaluation of global functioning was 
45.9 \pm 10.8 . All subjects were receiving antipsychotics, and the mean haloperidol equivalent dose was $17.4 \pm 11.9 \mathrm{mg} /$ day. Atypical antipsychotics were administered to 61 patients, typical antipsychotics to 28 patients, and both atypical and typical antipsychotics to 15 patients.

\section{Assessment}

Insight

Using the Japanese version ${ }^{17}$ of the SUMD ${ }^{1}$ insight was assessed by a research psychiatrist (YK) in a semistructured interview. The SUMD consists of general items to evaluate three general aspects of past and current insight into the illness, and subscale items to evaluate awareness and attribution of a maximum of 17 symptoms in the past and present. Both sets of evaluated items are rated on a 5-point scale (1-5), where higher scores indicate lesser insight into the illness. The general items consist of eight questions regarding present awareness and current awareness achieved through reflecting on the past in relation to awareness of the mental disorder, awareness of achieved effects of medication, awareness of need for medication, and awareness of the social consequences of the mental disorder. For the subscale items, we adopted six items used in the shortened version of the SUMD: ${ }^{18}$ hallucination, delusion, thought disorder, flat or blunted affect, anhedonia, and asociality. We asked participants to rate their awareness of such symptoms (awareness-subscale score). Thereafter, those who had a certain degree of awareness of symptoms (a score of $\leq 3$ ) were asked whether or not they thought the symptoms were attributable to a mental disorder (attribution-subscale score). The obtained scores were then used to calculate the current awareness-subscale score, past awareness-subscale score, current attribution-subscale score, and past attributionsubscale score. We also divided symptoms into positive (hallucination, delusion, thought disorder) and negative (flat or blunted affect, anhedonia, and asociality) in order to calculate the subscale score in each category.

\section{Subjective experience}

Various subjective experiences were evaluated using the following five questionnaires. First, subjective experience of deficits was evaluated by a research psychiatrist (YK) through interview questionnaires using the Japanese version $^{19}$ of the SEDS. ${ }^{13}$ This scale consists of 22 questions and covers a wide range of deficits of mental function. Each question is rated on a score of 0 (none) to 4 (severe), with higher scores indicating stronger subjective feelings of deficit. Questions are divided into five groups: abnormal thinking and concentration, disturbance of affect, impaired will and decreased energy, disturbance of perception, and intolerance of stress.

Subjective well-being was evaluated using the Japanese version $^{20}$ of the SWNS (Subjective Well-being under Neuroleptic drug treatment Short form). ${ }^{21}$ This is a self-reported 20-item questionnaire that evaluates the subjective wellbeing of patients undergoing antipsychotic therapy. Each item is rated on a 6-point Likert scale, where higher scores indicate better subjective well-being of the patient. It consists of 5 subscales: mental function, self-control, emotional regulation, physical functioning, and social integration.

Subjective QOL was evaluated using the Japanese version $^{22}$ of the Schizophrenia Quality of Life Scale (SQLS). ${ }^{23}$ This is a self-reported questionnaire consisting of 30 questions that evaluate the QOL of patients with schizophrenia. Each item is rated on a 5-point Likert scale, where a high score indicates low subjective QOL. It consists of the three subscales of psychosocial, motivation/energy, and symptoms/side effects.

Subjective symptomatic depression was evaluated with the Beck Depression Inventory (BDI). ${ }^{24,25}$ This is a selfreported questionnaire consisting of 21 items. The BDI is the most frequently used scale in screening and when evaluating the severity of symptomatic depression. Each item is rated on a 4-point Likert scale, where a high score indicates more severe depression.

Finally, attitudes toward medication were evaluated using the Japanese version of the Drug Attitude Inventory (DAI)-30, ${ }^{26}$ which together with the SWNS is often used for evaluating subjective response to drug therapy. The DAI-30 is a self-reported questionnaire consisting of 30 items, each of which is rated with a yes/no response. A high score indicates more positive attitudes toward medication. It also contains seven subscales, including subjective positive and subjective negative.

\section{Statistical analysis}

We first calculated raw scores for the SUMD and for the five measures of subjective experience. Thereafter, we analyzed the correlation between each item of the SUMD, and the correlation between each of the five measures of subjective experience using Spearman's rank correlation.

We then analyzed the relation between each of the measures and demographic variables (age at the time of survey, age at the onset of illness, sex, illness duration, duration of education, number of hospitalizations, and duration of hospitalizations) using Spearman's rank correlation and the 
Mann-Whitney $U$ test. These results were used to identify demographic variables that could represent confounding factors. Then, by defining those variables as control variables, partial correlations between the SUMD and the five measures of subjective experience were analyzed. Bonferroni correction was used for multiple comparisons, but to determine the relationship between insight and subjective experience widely, we paid attention also to correlations with a level of $P<0.05$.

\section{Results}

The mean scores of each item on the SUMD are shown in Table 1, and the mean scores of the five subjective experience measures are shown in Table 2. Table 3 shows correlations among the five subjective experience measures. Despite the relatively high correlations between each of the general items on the SUMD, no significant correlation was observed between the general items and the current awarenessand attribution-subscale scores. Awareness of mental disorder was relatively strongly correlated with the past awareness- and attribution-subscale scores $(r=0.30-0.52)$. With regard to the

Table I Scale to Assess Unawareness of Mental Disorder scores: means and standard deviation (SD)

\begin{tabular}{|c|c|c|}
\hline & Mean (range) & SD \\
\hline \multicolumn{3}{|l|}{ General items } \\
\hline \multicolumn{3}{|c|}{ Score on item I: awareness of mental disorder } \\
\hline Current illness & $1.8(I-5)$ & I.I \\
\hline Past illness & $1.4(I-5)$ & 0.9 \\
\hline \multicolumn{3}{|c|}{ Score on item 2a: awareness of achieved effects } \\
\hline \multicolumn{3}{|c|}{ of medication } \\
\hline Current illness & $\mathrm{I} .8(\mathrm{I}-5)$ & 1.0 \\
\hline Past illness & $1.9(1-5)$ & I.I \\
\hline \multicolumn{3}{|c|}{ Score on item $2 b$ : awareness of need for medication } \\
\hline Current illness & $1.7(I-5)$ & 1.0 \\
\hline Past illness & $1.5(1-5)$ & 0.9 \\
\hline \multicolumn{3}{|c|}{ Score on item 3: awareness of social consequences } \\
\hline \multicolumn{3}{|c|}{ of mental disorder } \\
\hline Current illness & $1.5(I-5)$ & 1.0 \\
\hline Past illness & $1.4(1-5)$ & 0.8 \\
\hline \multicolumn{3}{|l|}{ Subscale items } \\
\hline \multicolumn{3}{|l|}{ Awareness, total score } \\
\hline Current illness & $3.0(1-5)$ & 1.4 \\
\hline Current positive symptoms & $3.5(I-5)$ & 1.4 \\
\hline Current negative symptoms & $2.8(I-5)$ & 1.6 \\
\hline Past illness & $2.3(I-5)$ & 1.1 \\
\hline Past positive symptoms & $2.4(I-5)$ & 1.4 \\
\hline Past negative symptoms & $2.2(1-5)$ & 1.3 \\
\hline \multicolumn{3}{|l|}{ Attribution, total score } \\
\hline Current illness & $3.2(1-5)$ & 1.4 \\
\hline Current positive symptoms & $2.3(I-5)$ & 1.5 \\
\hline Current negative symptoms & $3.6(I-5)$ & 1.3 \\
\hline Past illness & $2.2(I-5)$ & 1.2 \\
\hline Past positive symptoms & $1.6(I-5)$ & 0.9 \\
\hline Past negative symptoms & $2.8(I-5)$ & 1.5 \\
\hline
\end{tabular}

Table 2 Subjective experience measures: means and standard deviation (SD)

\begin{tabular}{lll}
\hline & Mean (range) & SD \\
\hline SEDS & $20.3(0-56)$ & 13.9 \\
SWNS & $76.0(32-118)$ & 17.3 \\
SQLS & $47.8(3-93)$ & 18.6 \\
BDI & $14.5(0-44)$ & 10.2 \\
DAI-30 & $10.7(-20$ to 30$)$ & 12.6 \\
\hline
\end{tabular}

Abbreviations: SEDS, Subjective Experience of Deficits in Schizophrenia; SWNS, Subjective Well-being under Neuroleptic drug treatment Short form; SQLS, Schizophrenia Quality of Life Scale; BDI, Beck Depression Inventory; DAI, Drug Attitude Inventory.

subjective experience measures, there was a strong correlation between each of the four measures (SEDS, SWNS, SQLS, and BDI) ( $r=0.62-0.77)$, although the DAI-30 was weakly correlated with the other four measures $(r=0.17-0.32)$.

In terms of the relationship between demographic variables and each measure, duration of illness had a weak negative correlation with current awareness of need for medication on the SUMD ( $r=-0.28, P=0.017)$, a weak positive correlation with the past awareness-subscale score ( $r=0.38, P<0.001)$, and a weak positive correlation with the DAI-30 ( $r=0.27, P=0.022)$. Age at the time of survey had a weak negative correlation with current awareness of achieved effects of medication on the SUMD ( $r=-0.25, P=0.029)$, a weak negative correlation with current awareness of the need for medication ( $r=-0.31, P=0.008$ ), a weak positive correlation with the past awareness-subscale score $(r=0.29$, $P=0.016$ ), and a weak positive correlation with the past attribution-subscale score $(r=0.25, P=0.040)$. Total duration of hospitalization showed a weak positive correlation with the past awareness-subscale score on the SUMD $(r=0.34$, $P=0.004)$. There was a weak negative correlation $(r=-0.26$, $P=0.026$ ) between duration of education and current awareness of the need for medication on the SUMD. However, sex, age at the onset of illness, and number of hospitalizations were not significantly correlated with any measure.

Table 3 Spearman's rank correlations between subjective experience measures

\begin{tabular}{llllll}
\hline & SEDS & SWNS & SQLS & BDI & DAI-30 \\
\hline SEDS & & $-0.69^{\#}$ & $0.72^{\#}$ & $0.64^{\#}$ & $-0.24^{*}$ \\
SWNS & $-0.69^{\#}$ & & $-0.75^{\#}$ & $-0.62^{\#}$ & 0.17 \\
SQLS & $0.72^{\#}$ & $-0.75^{\#}$ & & $0.77^{\#}$ & $-0.32^{* *}$ \\
BDI & $0.64^{\#}$ & $-0.62^{\#}$ & $0.77^{\#}$ & & -0.23 \\
DAI-30 & $-0.24^{*}$ & 0.17 & $-0.32^{* *}$ & -0.23 & \\
\hline
\end{tabular}

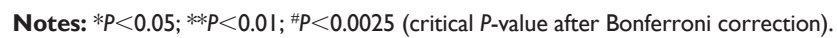
Abbreviations: SEDS, Subjective Experience of Deficits in Schizophrenia; SWNS, Subjective Well-being under Neuroleptic drug treatment Short form; SQLS, Schizophrenia Quality of Life Scale; BDI, Beck Depression Inventory; DAI, Drug Attitude Inventory. 
Among the demographic variables correlated with the SUMD and the five subjective experience measures, duration of illness and age at the time of survey showed a strong positive correlation (Pearson's $r=0.65, P<0.0001$ ), and duration of illness and total duration of hospitalization showed a moderate positive correlation (Pearson's $r=0.53$, $P<0.0001)$. Duration of education was independent of any other demographic variable. Therefore, we defined duration of illness and duration of education as control variables, and analyzed relationships between the SUMD and the five subjective experience measures using partial correlations (Table 4).

The SWNS exhibited a weak positive correlation with awareness of mental disorder and awareness of the social consequences of mental disorder on the SUMD ( $r=0.30$, $P=0.011$, and $r=0.27, P=0.022$, respectively). In other words, diminished subjective well-being was related to awareness of mental disorder and its social consequences. No significant correlation was observed between the SEDS, SQLS, and BDI and the general items $(r=0.02-0.23)$, although the SEDS and BDI showed a nonsignificant negative correlation with awareness of mental disorder on the SUMD $(r=-0.22, P=0.068$, and $r=-0.23, P=0.053$, respectively). The DAI-30 had a significant negative correlation with most general items on the SUMD ( $r=-0.41$ to -0.63 ), ie, positive attitudes toward medication were related to a high level of insight. Although the significant correlation observed between the DAI-30 and awareness of achieved effects of medication and need for medication on the SUMD was expected because of overlaps in contents and concepts, a significant correlation was also observed between the DAI-30 and awareness of past illness and the social consequences of mental disorder on the SUMD ( $r=-0.46$ and $r=-0.41,-0.54$, respectively). The four subjective experience measures apart from the DAI-30 showed a moderate-to-strong correlation with the current awarenesssubscale score $(r=0.50-0.68)$ and a weak correlation with

Table 4 Partial correlations between the Scale to Assess Unawareness of Mental Disorder and subjective experience measures covarying for duration of illness and education

\begin{tabular}{|c|c|c|c|c|c|}
\hline & \multicolumn{5}{|c|}{ Subjective experience measures } \\
\hline & SEDS & SWNS & SQLS & BDI & DAI-30 \\
\hline \multicolumn{6}{|l|}{ General items } \\
\hline \multicolumn{6}{|c|}{ Score on item I: awareness of mental disorder } \\
\hline Current illness & -0.22 & $0.30 *$ & -0.17 & -0.23 & $-0.35^{* *}$ \\
\hline Past illness & -0.11 & 0.20 & -0.10 & -0.10 & $-0.46^{\#}$ \\
\hline \multicolumn{6}{|c|}{ Score on item 2a: awareness of achieved effects of medication } \\
\hline Current illness & 0.10 & -0.05 & 0.04 & 0.15 & $-0.45^{\#}$ \\
\hline Past illness & -0.03 & -0.02 & 0.06 & 0.10 & $-0.50^{\#}$ \\
\hline \multicolumn{6}{|c|}{ Score on item $2 b$ : awareness of need for medication } \\
\hline Current illness & -0.13 & 0.12 & -0.07 & -0.08 & $-0.57^{\#}$ \\
\hline Past illness & -0.13 & 0.16 & -0.05 & -0.06 & $-0.63^{\#}$ \\
\hline \multicolumn{6}{|c|}{ Score on item 3: awareness of social consequences of mental disorder } \\
\hline Current illness & -0.08 & $0.27^{*}$ & -0.17 & -0.05 & $-0.4 I^{\#}$ \\
\hline Past illness & -0.13 & 0.20 & -0.13 & -0.09 & $-0.54^{\#}$ \\
\hline \multicolumn{6}{|l|}{ Subscale items } \\
\hline \multicolumn{6}{|l|}{ Awareness, total score } \\
\hline Current illness & $-0.68^{\#}$ & $0.60^{\#}$ & $-0.60^{\#}$ & $-0.50^{\#}$ & 0.08 \\
\hline Current positive symptoms & $-0.55^{\#}$ & $0.56^{\#}$ & $-0.48 * *$ & $-0.48 * *$ & -0.13 \\
\hline Current negative symptoms & $-0.64^{\#}$ & $0.57^{\#}$ & $-0.57^{\#}$ & $-0.45^{* * *}$ & 0.16 \\
\hline Past illness & $-0.26^{*}$ & $0.25 *$ & -0.17 & -0.19 & $-0.38^{* *}$ \\
\hline Past positive symptoms & -0.16 & 0.17 & -0.14 & -0.18 & $-0.38^{* *}$ \\
\hline Past negative symptoms & $-0.30 *$ & $0.33^{* *}$ & -0.21 & -0.18 & $-0.29 *$ \\
\hline \multicolumn{6}{|l|}{ Attribution, total score } \\
\hline Current illness & -0.15 & 0.08 & -0.05 & -0.14 & -0.12 \\
\hline Current positive symptoms & 0.03 & -0.01 & 0.16 & 0.07 & -0.02 \\
\hline Current negative symptoms & $-0.39 *$ & $0.36 *$ & $-0.38^{*}$ & $-0.36^{*}$ & 0.10 \\
\hline Past illness & 0.05 & -0.04 & 0.19 & 0.17 & $-0.42 * * *$ \\
\hline Past positive symptoms & 0.04 & -0.02 & 0.18 & 0.09 & $-0.33 *$ \\
\hline Past negative symptoms & -0.03 & 0.02 & 0.17 & 0.17 & $-0.32^{*}$ \\
\hline
\end{tabular}

Notes: $* P<0.05 ; * * P<0.01 ; * * * P<0.001 ; * P<0.0005$ (critical $P$-value after Bonferroni correction).

Abbreviations: SEDS, Subjective Experience of Deficits in Schizophrenia; SWNS, Subjective Well-being under Neuroleptic drug treatment Short form; SQLS, Schizophrenia Quality of Life Scale; BDI, Beck Depression Inventory; DAI, Drug Attitude Inventory. 
the subscale score of attribution of current negative symptoms $(r=0.36-0.39)$. This indicates a relationship between subjective distress and awareness of current symptoms, as well as between subjective distress and ability to attribute current negative symptoms to mental disorder. The SEDS and SWNS were weakly correlated with the subscale score of awareness of past negative symptoms on the SUMD ( $r=-0.30$ and $r=0.33$, respectively). The DAI-30 was not significantly correlated with the current awareness-subscale score or the current attribution-subscale score $(r=0.02-0.16)$ but had a weak-to-moderate negative correlation with the past awareness-subscale score $(r=-0.29$ to -0.38$)$ and the past attribution-subscale score $(r=-0.32$ to -0.42$)$. In other words, positive attitudes toward medication were related to awareness and attribution of past symptoms rather than awareness and attribution of current symptoms.

\section{Discussion}

Our study showed positive correlations between the SWNS, the awareness score of mental disorder, and the awareness score of the social consequences of mental disorder on the SUMD. Moreover, the SEDS and BDI were negatively (although not significantly) correlated with awareness of mental disorder. These results appear to indicate a relationship between awareness of subjective distress and awareness of having a mental disorder. The four measures of subjective experience - SEDS, SWNS, SQLS, and BDI - showed strong correlations with the current awareness-subscale score and weak correlations with the subscale score of attribution of current negative symptoms. Although it is understandable that the awareness of currently observed symptoms as abnormal is related to awareness of subjective distress, it was an unexpected finding that the ability to attribute negative symptoms to mental disorders was related to subjective experience. When patients could not attribute negative symptoms, such as flat or blunted affect, anhedonia, and asociality, to mental disorders, they most frequently attributed them to their original personality. Such patients are likely to have an asocial premorbid character, and they may therefore struggle to be aware of subjective distress. In contrast, patients who attribute negative symptoms to their mental disorders consider these symptoms as part of their illness rather than as an aspect of their personality, and they may relatively easily become aware of subjective distress.

With regard to the relationship between subjective well-being and insight, Valiente et $\mathrm{al}^{27}$ examined delusional patients, and showed that the group of patients with poor insight had better subjective well-being. Although this is consistent with the results of the present study, the subjects were evaluated using only item G12 of the PANSS, and no multidimensional evaluation was performed. Kim et $\mathrm{al}^{28}$ evaluated the subjective well-being and insight of patients prior to and 8 weeks after starting pharmacotherapy, and showed a correlation between improved insight and improved subjective well-being; however, evaluations in this study were also performed using only item G12 of the PANSS.

The relationship between subjective QOL and insight has been reported previously. Ritsner ${ }^{29}$ assessed 148 inpatients with schizophrenia using the self-reported questionnaire of the Insight $\mathrm{Scale}^{2}$ for evaluation of insight, and the Quality of Life Enjoyment and Satisfaction Questionnaire ${ }^{30}$ for longitudinal evaluation of subjective QOL. That study reported a relationship between increasing lack of awareness of mental illness and positive change in subjective QOL in several areas, although it found no relationship between change of attribution of symptoms to mental illness and change in subjective QOL. Doyle et $\mathrm{al}^{31}$ also assessed 40 outpatients to evaluate insight with the Insight Scale and subjective and objective QOL with several measures. They reported that objective QOL improved with insight; however, insight and subjective QOL were not related. Kao et $\mathrm{al}^{32}$ investigated the relationship between insight and subjective QOL in 104 chronic inpatients using the Self-Appraisal of Illness Questionnaire $^{33}$ and the World Health Organization Quality of Life-Brief Version. ${ }^{34}$ They observed that subjective QOL was better in patients with poor awareness of their need for treatment and of the presence and outcomes of their illness. Although these findings show some similarities to the relationship between the SUMD and SQLS scores found in our study, sufficient comparison could not be performed because of the different measures used.

With regard to the relationship between the SUMD and BDI scores, Bell et $\mathrm{al}^{35}$ found no significant correlation between general item scores on these two scales in 122 outpatients. Schwartz-Stav et $\mathrm{al}^{36}$ also studied 32 adolescent patients with schizophrenia; they found no significant correlation between BDI and general SUMD items but a strong correlation with subscale SUMD items. Cooke et $\mathrm{al}^{37}$ examined insight in 65 outpatients using the Schedule for the Assessment of Insight - Expanded ${ }^{38}$ and the Birchwood Insight Scale, ${ }^{2}$ and showed that factors including awareness of symptoms and awareness of issues were correlated with the BDI-II. Despite the differences in patient features between previous studies and our study, the results were similar in this regard. 
Unlike the other four measures of subjective experience, the DAI-30 had a significant negative correlation with most general items in the present study. This suggests a relationship between positive attitudes toward medication and awareness of having a mental disorder and its social consequences. These findings are consistent with those of previous reports. ${ }^{39-42}$ The DAI-30 is a questionnaire evaluating awareness of achieved effects of medication rather than awareness of subjective distress. Therefore, given the duplication of contents under awareness of achieved effects and need for medication on the SUMD, it is not unexpected that the DAI-30 was significantly correlated to both. Intriguingly, the DAI-30 did not correlate with the current awarenessand attribution-subscale scores, but correlated with the past awareness- and attribution-subscale scores. In order to have positive attitudes toward medication, it appears important not to be aware of current symptoms, but to recognize previous symptom exacerbations as abnormal and to attribute them to mental disorders. This could be a crucial point when undertaking psychoeducation.

Therefore, investigation from multiple viewpoints is necessary to elucidate the relationship between insight and subjective experience. Insight comprises many elements and is complicated by many factors. Subjective experience also has various concepts, although the importance of these concepts has not been fully established. Therefore, we believe that it is difficult to find a correlation between general insight and comprehensively assessed subjective experience. However, as shown in the present study, several scales related to subjective experience have been found to correlate with insight on a number of levels; awareness of subjective distress may thus be necessary to form insight. We believe that the degree of insight is determined on the basis of awareness that subjective pain differs qualitatively and quantitatively from a healthy state, as well as on the basis of such factors as psychotic symptoms, degree of cognitive impairment, and knowledge acquisition through psychoeducation.

The present study has some limitations. The first concerns the characteristics of patients recruited; only inpatients from a certain medical institution at the time of discharge were targeted, and more patients than anticipated were in the recovery phase after the first episode of psychosis. On the other hand, the study included few patients in the acute and chronic phases of mental disorder, and did not include outpatients or those under long-term hospitalization. Therefore, our results have limited generalizability to the overall population of patients with schizophrenia. However, for a study of inpatients from only one institution, we were satisfied with recruitment, because we surveyed $95 \%$ of patients (74 of 78) who were discharged within a certain period (ie, almost continuous sampling). The second limitation involves the evaluation methodology. Four of the five measures used for evaluating subjective experience employed self-report questionnaires, raising issues of reliability in this population. However, this is an issue faced by all researchers of subjective experience. Finally, our study was cross-sectional. As Kim et $\mathrm{al}^{7}$ noted, subjective experience in the past and its changes are important in relation to insight. However, very few longitudinal studies have been conducted, ${ }^{28}$ and the relationship between subjective experience and each level of insight has hardly been examined. It is unclear whether insight is formed by continuously feeling subjective pain or if the experience of alleviation of subjective pain leads to insight. Therefore, a longitudinal approach to the relation between insight and subjective experience is required for further study. We intend to follow the progress of the patients in this study and observe any changes in insight or subjective experience.

\section{Acknowledgments}

This research was funded by Grant-in-Aid for Young Scientists 18790818 (YK) from the Japanese Ministry of Education, Culture, Sports, Science and Technology (MEXT). We would like to thank Dr Yoshie Sakai, Dr Nobuki Kitagawa, Dr Kenzo Denda, and Dr Tsukasa Koyama for their valuable comments.

\section{Disclosure}

The authors report no conflicts of interest in this work.

\section{References}

1. Amador XF, Strauss DH, Yale SA, Flaum MM, Endicott J, Gorman JM Assessment of insight in psychosis. Am J Psychiatry. 1993;150(6): 873-879.

2. Birchwood M, Smith J, Drury V, Healy J, Macmillan F, Slade M. A self-report insight scale for psychosis: reliability, validity and sensitivity to change. Acta Psychiatr Scand. 1994;89(1):62-67.

3. David AS. Insight and psychosis. Br J Psychiatry. 1990;156(6): 798-808.

4. Markova IS, Berrios GE. The assessment of insight in clinical psychiatry: a new scale. Acta Psychiatr Scand. 1992;86(2):159-164.

5. McEvoy JP, Apperson LJ, Appelbaum PS, et al. Insight in schizophrenia. Its relationship to acute psychopathology. J Nerv Ment Dis. 1989;177(1): 43-47.

6. Peralta V, Cuesta MJ. A polydiagnostic approach to self-perceived cognitive disorders in schizophrenia. Psychopathology. 1992;25(5): 232-238

7. Kim Y, Sakamoto K, Kamo T, Sakamura Y, Miyaoka H. Insight and clinical correlates in schizophrenia. Compr Psychiatry. 1997;38(2):117-123.

8. Nakaya M, Kusumoto K, Ohmori K. Subjective experience of Japanese inpatients with chronic schizophrenia. J Nerv Ment Dis. 2002;190(2): 80-85. 
9. Süllwold L. Frankfurter Beschwerde-Fragebogen [Frankfurt Questionnaire of Complaints] (3). In: Süllwold L, Huber G, editors. Schizophrene Basisstörungen. Berlin: Springer; 1986:145-159. German.

10. Kay SR, Fiszbein A, Opler LA. The Positive and Negative Syndrome Scale (PANSS) for schizophrenia. Schizophr Bull. 1987;13(2): 261-276.

11. Gross G, Huber G, Klösterkotter J, Linz M. Bonner Skala für die Beurteilung von Basissymptomen [Bonn Scale for the Assessment of Basic Symptoms]. Berlin: Springer; 1987. German.

12. Jaeger J, Bitter I, Czobor P, Volavka J. The measurement of subjective experience in schizophrenia: the Subjective Deficit Syndrome Scale. Compr Psychiatry. 1990;31(3):216-226.

13. Liddle PF, Barnes TRE. The subjective experiences of deficits in schizophrenia. Compr Psychiatry. 1988;29(2):157-164.

14. Selten JP, Sijben NE, van den Bosch RJ, Omloo-Visser J, Warmerdam H. The subjective experience of negative symptoms: a self-rating scale. Compr Psychiatry. 1993;34(3):192-197.

15. American Psychiatric Association. Desk Reference to the Diagnostic Criteria from DSM-IV-TR. Washington: American Psychiatric Association; 2000.

16. American Psychiatric Association. Diagnostic and Statistical Manual of Mental Disorders. 4th ed. Washington: American Psychiatric Association; 1994

17. Sakai Y, Kim Y, Akiyama T, Kurita H. [Reliability and validity of the Scale to Assess Unawareness of Mental Disorder Japanese version (SUMD-J)]. Seishin Igaku. 2002;44(5):491-500. Japanese.

18. Amador XF, Flaum M, Andreasen NC, et al. Awareness of illness in schizophrenia and schizoaffective and mood disorders. Arch Gen Psychiatry. 1994;51(10):826-836.

19. Iwawaki A, Ohta K, Watanabe A, Narushima K. [The Japanese version of the Scale for the Assessment of Subjective Experience of Deficit in Schizophrenia (SEDS)]. Seishin Igaku. 1995;37(9):997-1003. Japanese.

20. Watanabe M, Matsumura H. [Reliability and validity of Subjective Well-being under Neuroleptic drug treatment Short form, Japanese version (SWNS-J)]. Jpn J Clin Psychopharmacol. 2003;6(7):905-912. Japanese.

21. Naber D, Moritz S, Lambert M, et al. Improvement of schizophrenic patients' subjective well-being under atypical antipsychotic drugs. Schizophr Res. 2001;50(1-2):79-88.

22. Kaneda Y, Imakura A, Ohmori T. [The Japanese version of the Schizophrenia Quality of Life Scale (JSQLS)]. Seishin Igaku. 2004;46(7): 737-739. Japanese.

23. Wilkinson G, Hesdon B, Wild D, et al. Self-report quality of life measure for people with schizophrenia: the SQLS. Br J Psychiatry. 2000;177(1): 42-46.

24. Beck AT, Rush AJ, Shaw BF, Emery G. Cognitive Therapy of Depression. New York: Guilford; 1979.

25. Beck AT, Ward CH, Mendelson M, Mock J, Erbaugh J. An inventory for measuring depression. Arch Gen Psychiatry. 1961;4(6):561-571.

26. Hogan TP, Awad AG, Eastwood R. A self-report scale predictive of drug compliance in schizophrenics: reliability and discriminative validity. Psychol Med. 1983;13(1):177-183.
27. Valiente C, Provencio M, Espinosa R, Chaves C, Fuentenebro F. Predictors of subjective well-being in patients with paranoid symptoms: is insight necessarily advantageous? Psychiatry Res. 2011;189(2): 190-194.

28. Kim JH, Ann JH, Lee J. Insight change and its relationship to subjective well-being during acute atypical antipsychotic treatment in schizophrenia. J Clin Pharm Ther. 2011;36(6):687-694.

29. Ritsner M. Predicting changes in domain-specific quality of life of schizophrenia patients. J Nerv Ment Dis. 2003;191(5):287-294.

30. Endicott J, Nee J, Harrison W, Blumenthal R. Quality of Life Enjoyment and Satisfaction Questionnaire: a new measure. Psychopharmacol Bull. 1993;29(2):321-326.

31. Doyle M, Flanagan S, Browne S, et al. Subjective and external assessments of quality of life in schizophrenia: relationship to insight. Acta Psychiatr Scand. 1999;99(6):466-472.

32. Kao YC, Liu YP, Chou MK, Cheng TH. Subjective quality of life in patients with chronic schizophrenia: relationships between psychosocial and clinical characteristics. Compr Psychiatry. 2011;52(2):171-180.

33. Marks KA, Fastenau PS, Lysaker PH, Bond GR. Self-Appraisal of Illness Questionnaire (SAIQ): relationship to researcher-rated insight and neuropsychological function in schizophrenia. Schizophr Res. 2000; 45(3):203-211.

34. [No authors listed]. Development of the World Health Organization WHOQOLBREF quality of life assessment. The WHOQOL group. Psychol Med. 1998;28(3):551-558.

35. Bell M, Fiszdon J, Richardson R, Lysaker P, Bryson G. Are self-reports valid for schizophrenia patients with poor insight? Relationship of unawareness of illness to psychological self-report instruments. Psychiatry Res. 2007;151(1-2):37-46.

36. Schwartz-Stav O, Apter A, Zalsman G. Depression, suicidal behavior and insight in adolescents with schizophrenia. Eur Child Adolesc Psychiatry. 2006;15(6):352-359.

37. Cooke M, Peters E, Fannon D, et al. Insight, distress and coping styles in schizophrenia. Schizophr Res. 2007;94(1-3):12-22.

38. Kemp R, David A. Insight and compliance. Chronic mental illness. In: Blackwell B, editor. Treatment Compliance and the Therapeutic Alliance. Vol 5. Newark (NJ): Gordon and Breach; 1997:61-84.

39. Rocca P, Crivelli B, Marino F, Mongini T, Portaleone F, Bogetto F. Correlations of attitudes toward antipsychotic drugs with insight and objective psychopathology in schizophrenia. Compr Psychiatry. 2008;49(2):170-176.

40. Robles García R, Salazar Alvarado V, Páez Agraz F, Ramírez Barret F. [Assessment of drug attitudes in patients with schizophrenia: psychometric properties of the DAI Spanish version]. Actas Esp Psiquiatr. 2004; 32(3):138-142. Spanish.

41. Freudenreich O, Cather C, Evins AE, Henderson DC, Goff DC. Attitudes of schizophrenia outpatients toward psychiatric medications: relationship to clinical variables and insight. J Clin Psychiatry. 2004;65(10):1372-1376.

42. Droulout T, Liraud F, Verdoux H. [Relationship between insight and medication adherence in subjects with psychosis]. Encephale. 2003; 29(5):430-437. French.
Neuropsychiatric Disease and Treatment

\section{Publish your work in this journal}

Neuropsychiatric Disease and Treatment is an international, peerreviewed journal of clinical therapeutics and pharmacology focusing on concise rapid reporting of clinical or pre-clinical studies on a range of neuropsychiatric and neurological disorders. This journal is indexed on PubMed Central, the 'PsycINFO' database and CAS,
Dovepress

and is the official journal of The International Neuropsychiatric Association (INA). The manuscript management system is completely online and includes a very quick and fair peer-review system, which is all easy to use. Visit http://www.dovepress.com/testimonials.php to read real quotes from published authors. 